\title{
Video Article \\ Nanomechanics of Drug-target Interactions and Antibacterial Resistance Detection
}

\author{
Joseph W. Ndieyira ${ }^{1}$, Moyu Watari ${ }^{1}$, Rachel A. McKendry ${ }^{1}$ \\ ${ }^{1}$ London Centre for Nanotechnology and Departments of Medicine, University College London
}

Correspondence to: Joseph W. Ndieyira at j.ndieyira@ucl.ac.uk

URL: https://www.jove.com/video/50719

DOI: doi:10.3791/50719

Keywords: Immunology, Issue 80, Engineering, Technology, Diagnostic Techniques and Procedures, Early Diagnosis, Bacterial Infections and Mycoses, Lipids, Amino Acids, Peptides, and Proteins, Chemical Actions and Uses, Diagnosis, Therapeutics, Surface stress, vancomycin, mucopeptides, cantilever sensor

Date Published: 10/25/2013

Citation: Ndieyira, J.W., Watari, M., McKendry, R.A. Nanomechanics of Drug-target Interactions and Antibacterial Resistance Detection. J. Vis. Exp. (80), e50719, doi:10.3791/50719 (2013).

\section{Abstract}

The cantilever sensor, which acts as a transducer of reactions between model bacterial cell wall matrix immobilized on its surface and antibiotic drugs in solution, has shown considerable potential in biochemical sensing applications with unprecedented sensitivity and specificity ${ }^{1-5}$. The drug-target interactions generate surface stress, causing the cantilever to bend, and the signal can be analyzed optically when it is illuminated by a laser. The change in surface stress measured with nano-scale precision allows disruptions of the biomechanics of model bacterial cell wall targets to be tracked in real time. Despite offering considerable advantages, multiple cantilever sensor arrays have never been applied in quantifying drug-target binding interactions.

Here, we report on the use of silicon multiple cantilever arrays coated with alkanethiol self-assembled monolayers mimicking bacterial cell wall matrix to quantitatively study antibiotic binding interactions. To understand the impact of vancomycin on the mechanics of bacterial cell wall structures ${ }^{1,6,7}$. We developed a new model ${ }^{1}$ which proposes that cantilever bending can be described by two independent factors; i) namely a chemical factor, which is given by a classical Langmuir adsorption isotherm, from which we calculate the thermodynamic equilibrium dissociation constant $\left(K_{d}\right)$ and ii) a geometrical factor, essentially a measure of how bacterial peptide receptors are distributed on the cantilever surface. The surface distribution of peptide receptors $(p)$ is used to investigate the dependence of geometry and ligand loading. It is shown that a threshold value of $p \sim 10 \%$ is critical to sensing applications. Below which there is no detectable bending signal while above this value, the bending signal increases almost linearly, revealing that stress is a product of a local chemical binding factor and a geometrical factor combined by the mechanical connectivity of reacted regions and provides a new paradigm for design of powerful agents to combat superbug infections.

\section{Video Link}

The video component of this article can be found at https://www.jove.com/video/50719/

\section{Introduction}

The molecular recognition paradigm underpins large swathes of biology and medicine. In pharmacology, for example the goal is to interfere with a pathological pathway by targeting a key participant of a biochemical process such as transglycoslyation or transpeptidation that catalyzes the crosslinking of bacterial cell wall peptides. The drug design is therefore reduced to defining a suitable molecule to target a specific bacterial docking site to induce a perfect fit. A classic example emulating the success of this approach is vancomycin (Van), which targets the peptidoglycan cell wall, a conserved structural feature of a bacterium. In a nascent Gram-positive bacterium, the peptidoglycan matrix consists of peptides terminating in the sequence Lysine-D-Alanine-D-Alanine, tethered via a C55 lipid linker ${ }^{8-10}$ here termed $\boldsymbol{D}$-Ala. These exposed peptides on the peptidoglycan precursors are fundamental for the mechanical protection of the bacterial cells against harsh environmental forces, and importantly, are not found in human cells, making them ideal targets for antibiotic drugs. Van specifically binds to the C-terminus of the bacterial cell wall peptides forming a moderately strong Van-peptide complex as shown in Figure 1. This interaction between Van and the exposed peptides blocks the actions of transpeptidases and transglycosylases, which catalyze the cross-linking of the cell walls ${ }^{1}$, effectively stopping them from crosslinking and therefore nanomechanically weakens the bacterial cell leading to its death by rupture ${ }^{1,6,7}$.

The emergence of vancomycin-resistant Enterococcus (VRE) is an increasing healthcare problem ${ }^{11}$ and because the bacterial resistance in Enterococci arises due to the subtle change of an amide linkage to an ester linkage ${ }^{8}$, this deceptively simple structural change at the surface of the bacterium deletes a single hydrogen bond from Van's docking site with subsequent modification of the binding pocket. Importantly this process alters the pentapeptide terminal alanine present in vancomycin susceptible Enterococcus (VSE) to a Lysine-D-Alanine-D-Lactate ${ }^{10,12}$ here termed $\boldsymbol{D}$-Lac, yielding a significant reduction in Van-D-Lac binding affinity by three orders of magnitude rendering Van therapeutically ineffective against Enterococcal infections ${ }^{1}$, The alarming growth of antibiotic-resistant bacteria is therefore driving the development of innovative approaches to accelerate and restore the antibacterial activity of antibacterial agents or the discovery of more powerful drugs against multidrug resistant bacterial infections. 
Our experiments using free-standing cantilevers is inspired by the systems such as cells which harness chemical energy into motion. The ability of cantilever sensors to translate chemical energy into mechanical motion makes them unique probes with considerable advantages for monitoring mechanical disruptions of bacterial cell wall targets in real time. The cantilever sensors require no reporter 'tags' and biomolecules are detected rapidly in a single-step reaction. They can screen multiple drugs in parallel under physiological environments such as buffered solutions and whole serum. In addition, the cantilever technology uses in situ referencing for differential measurements allowing them to specifically detect drug-target interactions and, by virtue of their fabrication via standard semiconductor processing routes, they are amenable for mass production and parallelization for high-throughput screening of thousands of drugs per hour. In particular multiple cantilever sensor arrays are useful tools for studying antibiotic resistance to vancomycin - because resistance to vancomycin is essentially a mechanical problem ${ }^{1,6,7}$. The reactions between a model bacterial cell wall target and Van in solution can be detected by monitoring the changes in clinically relevant stress induced from drug-target binding interactions. The generated stress from surface reactions which manifests itself in a cantilever bending signal is analyzed optically by illuminating sensors with a laser beam. Moreover by tailoring the receptive coating of bacterial surface target molecules on top of the cantilever sensor, close to an un-limited number of analytes (Van), the specific biochemical interactions and the biomechanics of model bacterial cell wall matrix is monitored in real time. Apart from the molecular recognition event, there are several factors that can cause cantilever sensors to deflect, which include - temperature variations, unspecific binding or changes of refractive index of the solution. To account for nonspecific signals, in situ differential measurements are performed where the bending of both the measuring and reference cantilevers are continuously monitored to analyze specific interactions. Furthermore, the detection sensitivities of cantilever sensors which depend on the surface chemistries and geometry can be enhanced by tuning the surface density $p$ (where $p$ is defined as the ratio of the surface area occupied by capturing molecules to the entire top surface area of the cantilever covered by the total population of molecules as determined by X-ray photoelectron spectroscopy (XPS) ${ }^{1}$.

Here this protocol details how vancomycin or any other antibiotic binding to bacterial cell wall precursor analogs (mucopeptides) at clinically relevant concentrations in buffered solution and blood serum can be detected by using label-free cantilever technology. Fresh gold surfaces are used because self-assembled monolayers (SAMs) tend to easily form stable layers on clean gold ${ }^{13,14}$. SAMs typically consists of short molecules with a thiol moiety covalently attached at the gold surface while the desired capturing unit on the other end is allowed to freely interact with the analyte targets in solution. Thiols form a flexible system particularly given that many thiol compounds with different chemical end groups are commercially available or are easily synthesized in a laboratory. However, care should be taken to ensure that molecules with a thiol moiety must have the correct end groups, for example in a protein or peptide conjugation, the amino group should face inwards for the reaction with the carboxylic group of the thiol moiety tethered on the surface to occur. Here, the thiols were directly conjugated with tripeptide mimetics of bacterial lipid II from the bacterial cell wall targets synthesized by solid phase methodology using commercially available preloaded Wang-D-Ala and Wang- $D$-Lac resins and the standard Fmoc-protecting group chemistry ${ }^{15}$. Although this description is focused to vancomycin, clearly it can be extended to other antibiotics and indeed further studies are invited by researchers from different fields particularly in biochemistry, pharmacology, and material science to easily adopt this protocol for their own experiments.

\section{Preparation of Cantilevers}

1. Using Teflon tweezers immerse selected number of cantilever chips (each cantilever measuring $500 \mu \mathrm{m}$ long, $100 \mu \mathrm{m}$ wide and $0.9 \mu \mathrm{m}$ thick) into a freshly prepared piranha solution (at ratio $1: 1 \mathrm{H}_{2} \mathrm{SO}_{4}$ and $\mathrm{H}_{2} \mathrm{O}_{2}$ ) for $20 \mathrm{~min}$.

2. After approximately 20 min remove the cantilever chips from piranha solution and rinse them thoroughly with deionized water and immediately transfer into a freshly prepared piranha solution. Repeat step 1.1 above if the chips contain spots of dirt otherwise proceed to the next step.

3. After a thorough cleaning in deionized water, rinse with pure ethanol and dry the cantilever chips on a hotplate at $75^{\circ} \mathrm{C}$ to remove any traces of water. Inspect them using an optical microscope to confirm their cleanliness.

4. Transfer the cleaned cantilever chips to an evaporation chamber and pump down, targeting to achieve a vacuum pressure of $10^{-7} \mathrm{mbar}$.

5. Once the required vacuum pressure is attained, coat one side of each cantilever array with a $2 \mathrm{~nm}$ titanium first to acts as a adhesion layer before an additional layer of $20 \mathrm{~nm}$ gold thick. To confirm their thickness, use quartz crystal monitor placed directly above the target source.

6. Leave gold coated cantilever sensor chips in the chamber for 1-2 hr to cool under vacuum before opening.

7. Transfer the freshly evaporated chips to a vacuum storage vessel filled with argon to prevent any form of contaminations.

\section{Cantilever Chip Functionalization}

1. First, arrange micro-capillary tubes on a functionalization stage ${ }^{2}$ according to the cantilever pitch size of $250 \mu \mathrm{m}$.

2. Then inject $2 \mathrm{mM}$ ethanolic thiol solutions of surface target molecules, i.e. bacterial mucopeptides (D-Ala and $\boldsymbol{D}$-Lac), and an 'inert' alkanethiol terminating in triethylene glycol (PEG) known to resist biomolecular adsorption. ${ }^{16}$ Each of the capillary tubings should contain an assorted surface capture molecules determined randomly to avoid user bias.

3. Next incubate for $20 \mathrm{~min}$ the cantilevers in the microcapillaries filled with thiol solutions containing surface capturing molecules. Ensure that the solutions of the surface capture molecules are confined onto each individual cantilever sensor to avoid or minimize cross-contaminations. If this process is employed correctly, it should systematically alter the freshly prepared gold coated cantilevers into chemically active sensors formed by allowing SAMs of bacterial mucopeptide targets to form on gold coated surfaces as receptors.

\section{Solution Preparations}

1. Dissolve $0.1 \mathrm{M}$ mono- and di-basic sodium phosphate salts in ultrapure water $(18.2 \mathrm{M} \Omega \cdot \mathrm{cm}$ resistivity) and mix to yield a pH value of 7.4 .

2. Add $0.002 \%$ of PS 80 to the buffered solutions to minimize aggregation effects caused by nonspecific interactions of drug molecules to the glassware.

3. Dilute drugs with freshly buffered solutions to the desired different concentrations that will enable calculation of $K_{d}$. 
4. Filter the freshly prepared drug solutions using $0.2 \mu \mathrm{m}$ filters and sonicate for $5 \mathrm{~min}$ at room temperature before purging with argon.

5. Repeat the procedure with drug in buffered serum using whole serum. Gently vortex for 15 min with additional 5 min to ensure complete solubility.

\section{Surface Stress Detection}

1. Load a functionalized cantilever sensor chip into a liquid flow cell chamber.

2. Align the laser spot onto the free end of each sensor and confirm the alignment by heating up the liquid chamber for $1^{\circ} \mathrm{C}$. All eight gold coated cantilever sensor arrays should undergo compressive downward bending because of the bimetallic effect caused by the differences in expansion rates of silicon and gold.

3. After heating for approximately $10 \mathrm{~min}$, allow the cantilevers to cool for further $10 \mathrm{~min}$

4. Calculate the bending variation at the maximum bending signals between individual cantilever sensors and if the relative standard deviation of the bending signals is $\leq 5 \%$ then accept the alignment as desirable otherwise repeat the process.

5. Next, measure the resonant frequencies of all eight cantilevers to calculate their spring constants. If the variation of the spring constant between each cantilever sensor within a chip is $\leq 1 \%$ then accept the chip as having consistent mechanical properties otherwise replace the cantilever chip sensor.

6. Next, use a fluidic system (Model Genie Plus) via a six-way valve to achieve the liquid exchange within the flow cell while data acquisition should be achieved using an automated LabView software.

7. To monitor the cantilever bending data, use the following measurement protocols: i) inject either buffer solution or serum without drug for control measurement lasting for 5-30 min to establish a baseline; ii) inject drug solution for 30-60 min; iii) inject $10 \mathrm{mM} \mathrm{HCl}$ wash for $10-60$ min to dissociate bound drug complex; iv) finally inject a further washing step using buffer solution for another 5-30 min to regenerate the surface peptides and to restore the baseline signal. Always ensure that all signals are acquired under constant liquid flow rate of $30-180 \mu \mathrm{l} /$ min and at fixed temperature of $25^{\circ} \mathrm{C}$ in a temperature-controlled cabinet.

8. The absolute bending signals of all eight cantilevers should be monitored using serial time multiplexed optical beam method with a single position sensitive detector.

9. To analyze the bending signals from each concentration of drug, the resulting differential bendings are converted into a differential stress between the upper and lower sides of the cantilever using Stoney's equation.

\section{Representative Results}

The stress change measured using nano-scale precision with unprecedented sensitivity to a single $\mathrm{H}$-bond deletion, is exploited to track the disruptions of model bacterial cell wall biomechanics in real time (Figures 2a-d). The limit of drug detection sensitivity for Van was investigated by serially diluting its concentrations in steps from $1,000 \mu \mathrm{M}$ to $\leq 10 \mathrm{nM}$, revealing $10 \mathrm{nM}(\sim 15 \mathrm{ng} / \mathrm{ml})$ as the lowest detectable concentration giving rise to an average of $\sim-9 \pm 2 \mathrm{~nm}$ as the differential bending signals (Figure 2c). The ability to detect antibiotics under physiological environment was further investigated in serum at a clinically relevant concentration range of 3-27 $\mu \mathrm{M}^{17}$. Upon injection of $7 \mu \mathrm{M}$ Van in serum ( $90 \%$ fetal calf serum plus $10 \%$ sodium phosphate buffer $\mathrm{pH} 7.4$ ) across all cantilevers under identical conditions, the differential signal for $\boldsymbol{D A} \boldsymbol{A} \boldsymbol{a}$ in serum was $105 \pm 4 \mathrm{~nm}$ while for DLac coated cantilevers, no bending was observed (Figure 3 ). The considerable bending signals observed for DAla coated (vancomycin susceptible) cantilevers are caused by the strong drug-target binding interactions. However for DLac coated (vancomycin resistant) cantilevers, the presence of ground-state repulsion of the oxygen lone pair and the reduced $\mathrm{NH}$ bond in the vancomycin binding pocket ${ }^{10,12}$ contributes to the weakening of the drug-target binding interactions which results in less or no cantilever bending, particularly for lower vancomycin concentrations (Figure 3). However, for high vancomycin concentration, we observe measureable bending signals for DLac. Furthermore, our experimental design is important for in situ referencing where all cantilevers coated with both DAla and DLac are simultaneously exposed to the same analyte in real time.

To understand the precise role of chemistry and geometry, we designed a model ${ }^{1}$ describing the correlation between solvent interactions and surface mechanics. This produces the surface stress:

$$
\Delta \sigma_{\text {eq }}=\frac{a \cdot[\operatorname{Van}]}{K_{\mathrm{d}}+[\operatorname{Van}]}\left(\frac{p-p_{\mathrm{c}}}{1-p_{\mathrm{c}}}\right)^{\alpha} \text { for } p>p c \text { and zero otherwise (1) }
$$

The first term in equation (1) is the Langmuir Adsorption Isotherm, accounting for drug-target binding events and the second term is the power law form describing the large-scale mechanical consequences of stressed network formation. The constant a corresponds to maximum surface stress when all the accessible binding sites are occupied and $K_{d}$ is the surface equilibrium dissociation constant on the cantilever. The analysis as shown in Figure 4 is the outcome of the global fit of equation (1) superimposed onto measured differential stress signals revealing; a $\sim 29.7 \pm 1.0$ or $14.1 \pm 3.0 \mathrm{mN} / \mathrm{m}$ and $K_{d} \sim 1.0 \pm 0.3$ or $800 \pm 310 \mu \mathrm{M}$ for $\boldsymbol{D}-\boldsymbol{A l a}$ or $\boldsymbol{D L a c}$ peptides, where a corresponds to a measure of surface stress when all the accessible binding sites are occupied. The enhanced sensitivity of cantilever was tuned by systematically varying the peptide densities $p$ while monitoring the stress data as a function of $p$ while antibiotic concentrations fixed at 10, 100, and $250 \mu \mathrm{M}$, respectively (Figure 5). The process was repeated with stress as a function of Van concentrations while the peptide densities fixed at $p \sim 100 \%$. 


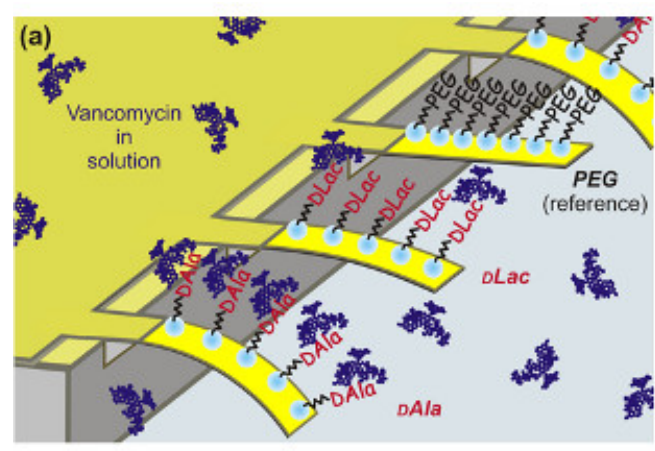

(b)
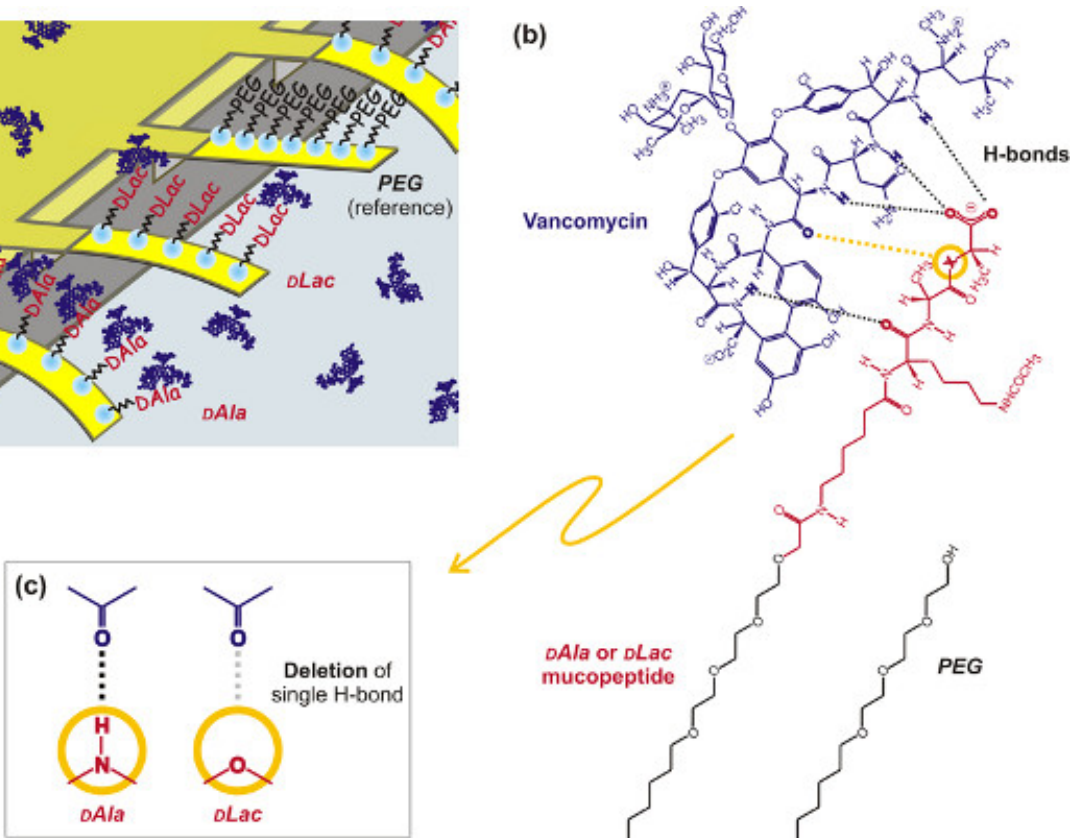

Vancomycin
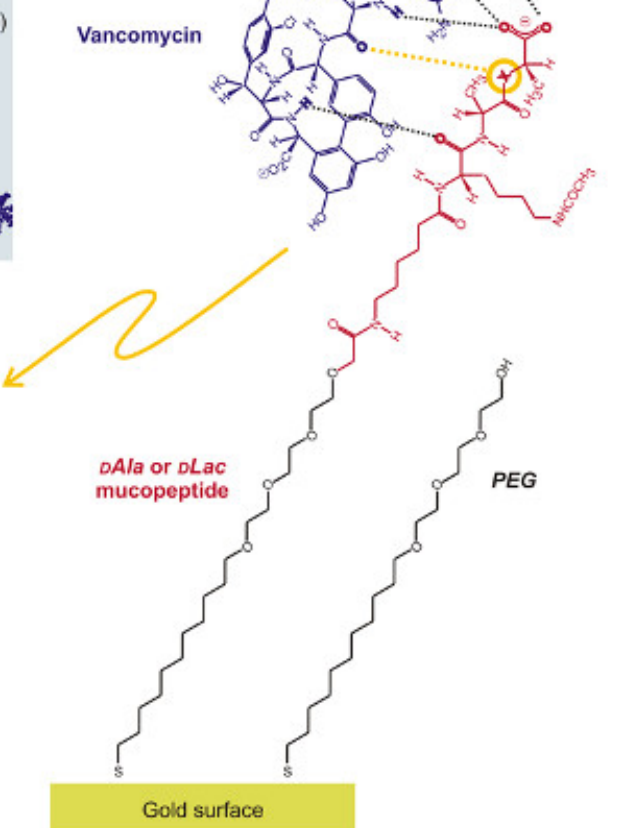

Figure 1. Cantilever detection of antibiotic surface binding interactions. a) Schematic representation of a cantilever sensor array and the mode by which the drug-target interactions are detected nanomechanically. b) Chemical binding interaction between a drug molecule (Van) and the bacterial mucopeptide analog (D-Ala or $\boldsymbol{D}$-Lac). c) The mechanism by which a mutated vancomycin susceptible bacteria (VSE) acquires resistance to vancomycin by deleting a single $\mathrm{H}$-bond in the binding pocket. Click here to view larger figure. 
a)

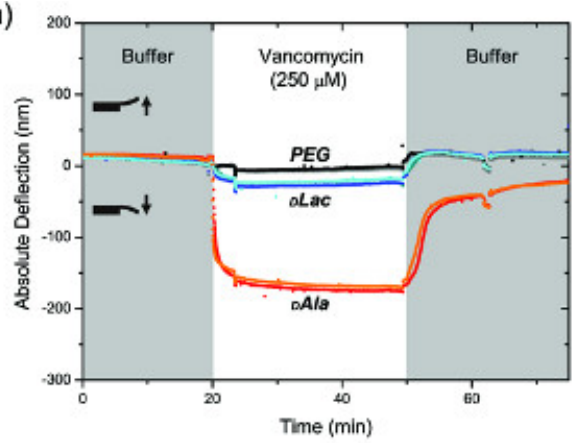

c)

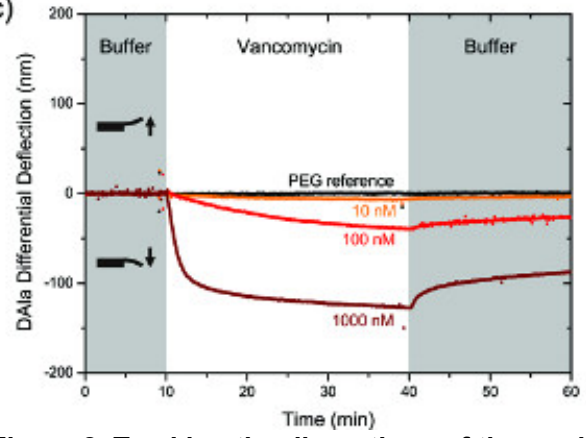

b)

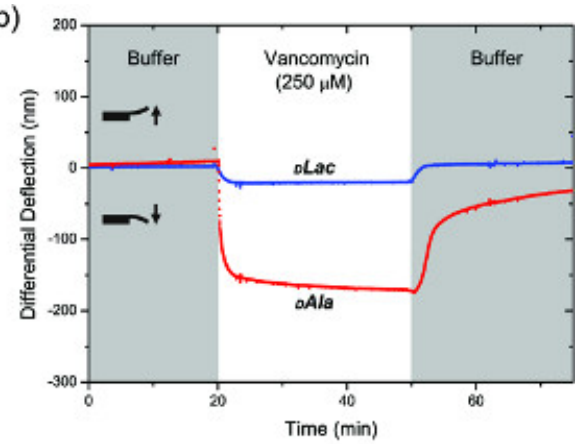

d)

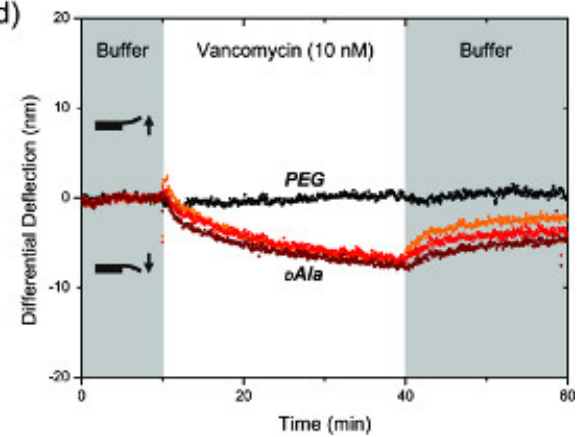

Figure 2. Tracking the disruptions of the model bacterial cell wall biomechanics in real time. a) Absolute bending signals of $D$-Ala , $D$ Lac and PEG coated cantilevers in phosphate buffer and $250 \mu \mathrm{M}$ vancomycin. The differential PEG reference signals are shown in black lines.

b) Differential bending signals of $\boldsymbol{D}$-Ala and $\boldsymbol{D}$-Lac coated cantilevers in phosphate buffer and $250 \mu \mathrm{M}$ vancomycin. c) The differential cantilever deflection of a dose dependent signal as a function of time in the presence of vancomycin concentrations in the order of $10 \mathrm{nM}$ (yellow line), 100 $\mathrm{nM}$ (red line) and 1,000 $\mathrm{nM}$ (dark red line) respectively. d) The differential cantilever deflection signals for three $\boldsymbol{D}$-Ala coated cantilever sensors as a function of time in the presence of $10 \mathrm{nM}$ vancomycin. Click here to view larger figure.

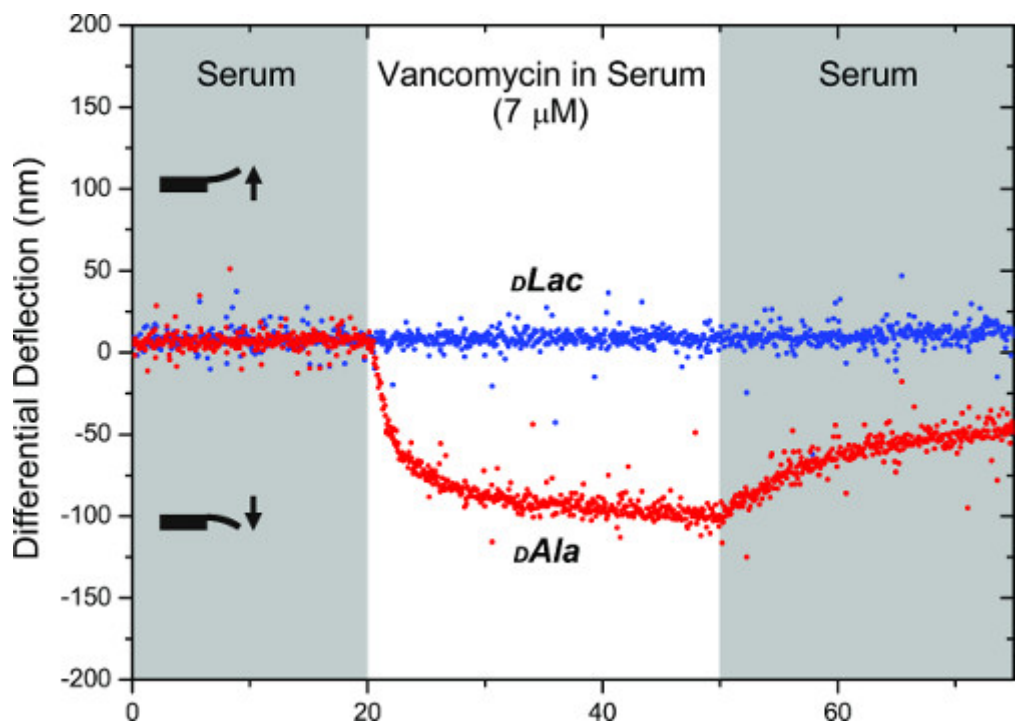

Figure 3. Tracking of the disruptions of the model bacterial cell wall biomechanics in real time for a $D$-Ala and $D$-Lac coated cantilevers in the presence of $7 \mu \mathrm{M}$ vancomycin in calf fetal serum. 


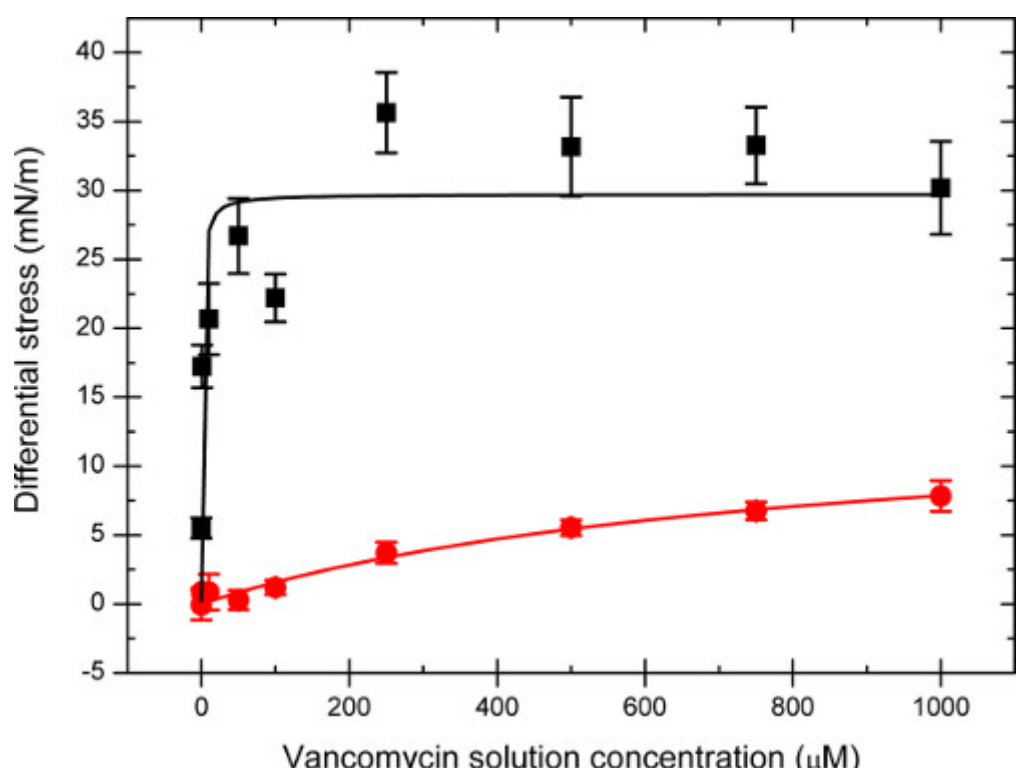

Figure 4. Investigating the nanomechanics of drug-target interactions. Plot showing the measured differential surface stress response for $\boldsymbol{D}$-Ala (black circles) and $\boldsymbol{D}$-Lac (red circles) coated cantilevers as a function of vancomycin concentration in solution [Van]. The data are described by equation (1) (solid lines). ${ }^{1}$

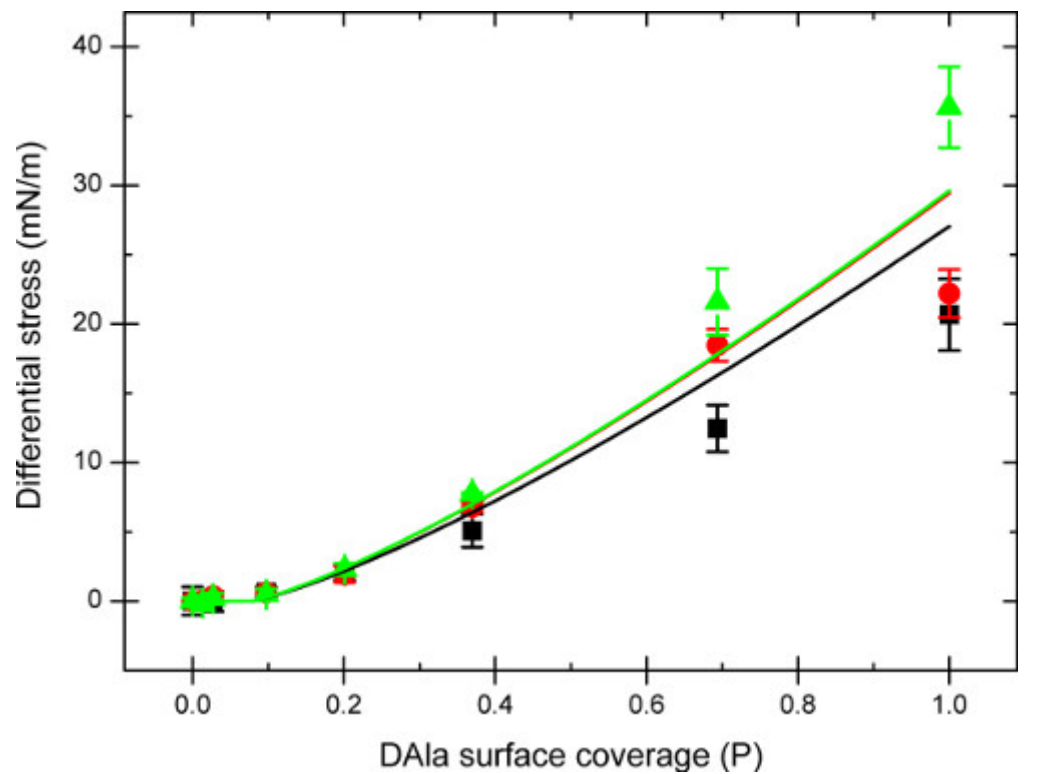

Figure 5. Optimization of nanomechanical detection sensitivity of receptor-ligand interactions by investigating the dependence of receptor loading and geometry via mixed monolayers. Measured differential surface stress responses of cantilever sensors as a function of $D$-Ala surface coverage, $p$ in the presence of fixed vancomycin concentrations in solution at $10 \mu \mathrm{M}$ (black line), $100 \mu \mathrm{M}$ (red line), and $250 \mu \mathrm{M}$ (green line).

\section{Discussion}

These results demonstrate that cantilever array sensors have the sensitivity to detect and quantify changes in drug-target binding interactions especially in vancomycin resistance associated with the deletion of a single H-bond from the drug's binding pocket. We show a nanomolar detection sensitivity of Van in agreement with previous Surface Plasmon Resonance (SPR) studies ${ }^{18,19}$, and reveal that the cantilever method can directly detect and quantify drug molecules in blood at clinically relevant concentrations as routinely applied in clinical practice. Our data suggest that the differential surface stress can be described by a product form equation (1), i.e. (i) a chemical term describing the specific drugtarget binding events, and (ii) a geometrical term describing the mechanical connectivity between chemically reacted surface sites, implying that the local chemical interactions decouple from the global mechanical interactions of the cantilever. While the chemical term is given by the classical Langmuir adsorption isotherm, the geometrical term reveals a percolative mechanism of the drug-target induced surface stress changes. The critical threshold pc $\sim 10 \%$ (Figure 5) was necessary to detect the differential cantilever bending, showing that surface stress is transduced collectively when a relatively large surface fraction is occupied by antibiotic molecules. For $p \geq p c$, the mechanical connectivity between chemically transformed surface sites is gradually established, and the short-range repulsive interactions such as steric interactions between the nodes of the nanomechanical network gives rise to increasing downward bending of the entire cantilever. It is speculated that our 
nanomechanical percolation model may play an important role in the glycopeptide antibiotic mode of action in real bacteria. These findings highlight the high sensitivity of cantilever technology for studying antibiotics' mode of action and represent a novel research tool for studying drugs to improve understanding of the operations of antibiotics at the nano-scale to inform and enable discovery of powerful drugs to control the problems of superbug infections. To prepare the cantilever system for reproducible and sensitive measurements, we have addressed a set of targets in the protocol, particularly for sample loading in microfluidic cell and standard operating procedures to allow quantitative nanomechanical detections.

\section{Significance of cantilever technology with respect to existing methods}

In summary we point out that while this technology was proposed more than 25 years ago, it has not found its way into the clinic because of lack of careful and repetitive measurements on medically relevant targets. Here we demonstrate the procedures that establish the relevance of using nanomechanical cantilevers to investigate the mechanical influence of antibiotics on the bacterial cell wall targets and to detect antibacterial resistance. Conventional drug screening methods require some type of fluorescent or radioactive labeling of a reporter molecule to measure the binding of an analyte to its target, often in connection with a competitive or enzymatic binding assay and protein assays ${ }^{20}$. Labeling of biomolecules is not only time-consuming and expensive but the label can also interfere with the molecular interaction by obstructing the binding site, leading to false negatives. In addition, fluorescent compounds are often hydrophobic which can lead to background binding and false positives. Due to these limitations, there is an increasing interest in novel label-free techniques that allow virtually any molecular complex to be screened with minimal assay development. The most established label-free surface technologies at present are SPR and Quartz Crystal Microbalance (QCM). In contrast to SPR that measure the dielectric constant, a label - free cantilever technology detects the surface strain generated by a ligand-receptor interaction, which can directly measure the nanomechanical forces generated by the specific binding of ligands to surface receptors. The uniqueness of these sensors is that their sensitivity does not rely on dielectric properties caused by mass change due to the analyte binding as in SPR and QCM but rather on a minutest induced change in in-plane surface stress, making the technology uniquely suited to study the nanomechanics of drug molecules at clinically relevant antibiotic concentrations $(3-27 \mu \mathrm{M})^{17}$. Cantilevers are also particularly well suited to small molecule (such as DNA fragments and drugs) detection under physiological conditions including complex environments which is largely the basis of the pharmaceutical industry and will therefore serve as a complementary tool in drug discovery. Cantilever technology have successfully been applied in the fields of genomics ${ }^{3,5}$, gas sensing ${ }^{21}$, proteomics ${ }^{22}$, and drugs ${ }^{1}$. Moreover, cantilevers are fabricated using low cost silicon technology and due to their compatibility with microfabrication processes, cantilevers can be miniaturized for improved sensitivity and parallelization into large arrays of sensors for multiple drug compound screening and higher throughput information-rich screening assays. Improvements of the instrumentation and experimental design will allow a wide variety of interactions to be analyzed in real time to help advance the search for a new generation of superdrugs to tackle the problems of multidrug resistant infections.

\section{Critical steps within the protocol}

The development of robust measurement protocols is central to the applications of this technology. To achieve satisfactory quantitative drugtarget measurements and to determine the lowest concentration of antibiotics that could be detected in buffer or blood serum, critical steps within the protocol were addressed. The first task involves tuning and optimizing surface capture chemistries to enhance cantilever detection specificity and sensitivity. Undeniably, surface stress transduction is a collective phenomenon, requiring a relatively large fraction of the surface to be covered to establish connectivity between chemically reactive regions. We show that by varying the density of the underlying surface peptides, a critical threshold $\sim p \geq 10 \%$, is determined where the surface stress scale as a function of peptide density and zero otherwise (Figure 5). It is important that experiments are designed to investigate the uniformity of stress along the cantilevers to ensure maximum signal deflections for sensitive measurements. In addition, efficient surface regeneration protocols need to be in place, thereby allowing multiple cycle measurements and to reduce costs for each test. While designing a receptor surface using thiolated hydrophobic end, orientation of the receptor molecule and spacing between them is vital to allow self-assembly of dense packing due to Van-der-Waals interactions between the molecules to minimize nonspecific interactions. Furthermore, the capture molecules should contain a polyethylene glycol (PEG) linker to allow some part of the sensing matrix to be hydrophilic to prevent insertion of molecules in the analyte solution from reacting directly with the uncoated gold surface. The PEG linker molecules must act as spacers to reduce steric constraints and therefore allow the solution analytes to interact specifically with the surface receptors to induce measureable surface stress change. The cantilever array sensor must be assayed with at least an in situ reference measurement and the signal displayed in real-time is a differential deflection, obtained by subtracting the absolute deflection of the reference cantilever from the sensing cantilevers. Thus a reference cantilever is essential to account for nonspecific interactions such as temperature changes, changes in refractive index or interactions on the nonfunctionalized underside of the cantilever. Optimization of the flow rate $(\sim 30-150$ $\mu \mathrm{l} / \mathrm{min}$ ) forms a critical step within the protocol because it ensures the efficient exchange of liquids and a sufficient steady mass transport of solution materials. The design of a liquid cell must allow optimum volume (5-80 $\mu \mathrm{l})$ for fast flow rates to enable perfect liquid exchange to overcome mass transport limitations. The flow rate is especially critical while performing kinetic measurements ${ }^{23}$. Large volume liquid chambers require uncontrollable high flow rates leading to large sample volume requirements, which unnecessarily increases the price of the assay. Previously we have used gravity flow to inject different samples into the measurement liquid chamber. Gravity flow has the advantage that it does not require any mechanical parts and therefore does not introduce additional noise into the system. However, its significant disadvantage is that it only works reliably at relatively high flow rates $(\sim 200 \mu \mathrm{l} / \mathrm{min})$. Obviously, a lower flow rate $(\leq 1 \mu \mathrm{l} / \mathrm{min})$ would require limited sample volumes per unit time but on the other hand it makes the reactions much slower and hence requires longer contact times. Moreover, gravity flow has a large variance in its flow rate as it depends on the height difference between the inlet and outlet, which decreases as the sample solutions are consumed during the experiment. To avoid gravity flow problems, a syringe pump should be used. The advantage of using a syringe pump is that it allows a constant flow rate over a long period of time allowing the experiments to be realized in a more controlled environment.

\section{Limitations of the protocol}

The major challenge in obtaining a reproducible and specific biological detection using cantilever sensors lies in ensuring that the properties of the receptor layer are biochemically "active" and uniform for each and every assay. The secret to experimental success is in a careful pretreatment of the sensor chip with a standardized cleaning and immobilization protocol with linker chemistries to orient the receptor molecules in their active conformation. In our current setup, we functionalize cantilever arrays using small glass capillaries, which could be subject to some drawbacks and can be problematic in some cases. These glass capillaries are open at both ends and therefore the sample solvents can evaporate easily. For example, if the temperature of the functionalization stage is not accurately controlled, the evaporation rate can vary significantly at different times particularly when using volatile solvents like ethanol. There is also a possibility of slight variation in the 
incubation time from one cantilever to another given that the sensing liquids have to be loaded consecutively into the capillaries. The other limiting factor in capillary functionalization is the lack of the ability to ensure that cantilevers are always inserted into the capillaries in exactly the same way. In addition, sometimes the cantilevers have to be pulled out slightly from the capillaries in order to prevent cross-contamination as the liquid samples can flow onto the chip body. We can solve these problems by employing inkjet spotters as an alternative surface coating procedure to coat cantilevers. While it would allow the exact control of sample coating with the possibility of scaling up for large arrays, the most common drawback is that the small drops that are deposited onto the cantilevers can evaporate within seconds and requires a controlled humidity environment. Therefore, the incubation time cannot be adjusted easily, which might be desirable for some applications. The subtle interplay of the factors such as sample volume, incubation time and evaporation rate have direct impact on the exposure of the cantilevers to the functionalization sample and care must be taken to ensure optimized surface chemistries for cantilever assays as any small variation in the receptor molecular density will have a large effect on the cantilever response.

\section{Experimental design modifications (i.e. alternative techniques or materials)}

To overcome the major challenge in obtaining reproducible coating procedure for the future development of the cantilever technology, different strategies are required that would use cantilevers integrated in microfluidic channels. The idea is that special cantilever chips should be designed so that each cantilever is placed into its own channel to allow online in situ functionalization procedures where the channels are addressed individually. These experimental design modifications would allow coating process to be performed in a controlled and closed environment where the exposure to the solvent is precisely monitored by the incubation time and flow rate for automated immobilization of capture molecules on cantilever chips. The same channels could then be used for the actual binding experiments where all cantilevers could be exposed to the same analyte solution. Besides the cantilever functionalization procedure, the cantilever readout mechanism would also need improvement. The optical beam deflection method is highly sensitive and it has been used successfully in Atomic Force Microscopy (AFM) technology for many years. Nevertheless, for the free standing cantilever sensor applications the optical readout has some drawbacks, for example it does not allow measurements in opaque liquids such as blood, the alignment of an array of lasers can be time-consuming and tedious and the current configuration cannot differentiate between tilting and vertical deflections. Thus the future development of the cantilever technology will have to consider aspects of the general sensor design (e.g. cantilever geometries) and the cantilever readout for robust measurement protocols both in the fields and laboratory environments. Manalis and coworkers ${ }^{22}$ have developed novel types of hollow cantilever sensors where the cantilevers have an embedded microfluidic channel inside the beam, allowing the device to be operated in a vacuum with high-quality factors. In this mode, cantilevers act as microbalance ${ }^{22}$, and therefore it is no longer necessary to have an asymmetry between the two sides with respect to functionalization, thus the capture molecules can be physisorbed or covalently attached directly on the silicon, typically using SAMs or silanes ${ }^{24}$. The cantilevers can also be modified with a thin polymer layer which is then conjugated to antibodies ${ }^{25}$ for biochemical sensing.

\section{Troubleshooting}

A common problem associated with the cantilever measurements is the introduction of air bubbles into the microfluidic flow cell. Care must be taken to ensure that no air bubbles are introduced into the liquid cell while mounting the chip. Signal drifting is also another common problem caused by the differences in the temperature of samples. The cantilevers must be equilibrated to stabilize before measurements are undertaken. All the buffers, analytes and regeneration solutions must be stored in the same room as the cantilever instrument to allow all the solutions to have the same temperature. Although the syringe pump can provide very accurate and extremely low flow rates, it is generally associated with mechanical noise in the cantilever measurements. It is therefore important to devise a simple and effective noise reducer to avoid unnecessary noise in the deflection signals. The noise reducer consists of small liquid reservoir located between the syringe pump and the liquid cell, which absorbs the mechanical noise from the pump. Because of the sensitive nature of optical detector, the cantilever measurement system should ideally be operated in an enclosed setup to block any stray lights from interfering with the optical readout system. In addition, the quality of the sensing layers can decrease significantly if a large number of washing steps are performed on the cantilevers limiting the lifetime of the sensor chip.

\section{Alternative or future applications after mastering this technique}

Cantilevers coated with SAMs terminating in an amino- or carboxyl-group can be used as $\mathrm{pH}$ sensors. The functional end groups protonate or deprotonate depending on the $\mathrm{pH}$ of the solution and can generate a surface charge that leads the cantilever to bend ${ }^{24}$. Given that the cantilever sensors can track drug-target interactions associated with the destabilization of the cell wall of life bacteria ${ }^{1,6,7}$, they will therefore help in the search and for the development of a new generation of antibiotics to combat drug-resistant infections. In future cantilevers would be used as microbalances to measure the mass and growth rates of single cells ${ }^{26}$. The cantilever technology will prove beneficial to the study of cellular responses to different growth factors or drugs. It will also offer a novel tool for rapid detection of multiple biomarkers, which has immediate relevance in medical and point-of-care applications. Given the versatility, small size, and robustness of cantilever sensors, they will form a sensitive monitor of harmful environmental factors. They have already demonstrated their sensitivity in detecting toxic and harmful gases that can escape from the laboratory and industrial production units into the environment, such as hydrofluoric acid ${ }^{27}$ or hydrogen cyanide ${ }^{28}$.

\section{Disclosures}

No conflicts of interest is declared

\section{Acknowledgements}

Joseph W. Ndieyira was supported by Engineering and Physical Sciences Research Council (EPRSC), Interdisciplinary Research Centre in Nanotechnology (IRC), the Royal Society (RS) and Biano consultancy (BNC). We thank, Alejandra Donoso Barrera, Dejian Zhou, Manuel Vögtli, Matthew Batchelor, Matthew A. Cooper, Torsten Strunz, Trevor Rayment and Gabriel Aeppli for helpful discussions. 


\section{References}

1. Ndieyira, J.W. et al. Nanomechanical detection of antibiotic-mucopeptide binding in a model for superbug drug resistance. Nat. Nanotech. 3 , 691-696 (2008).

2. Zhang, J. et al. Rapid and label-free nanomechanical detection of biomarker transcripts in human RNA. Nat. Nanotech. 1, 214-220 (2006).

3. McKendry, R.A. et al. Multiple label-free biodetection and quantitative DNA-binding assays on a nanomechanical cantilever array. Proc. Natl. Acad. Sci. U.S.A. 99, 9783-9788 (2002).

4. Wu, G. Datar, R.H., Hansen, K.M., Thundat, T., Cote, R.J., \& Majumdar, A. Bioassay of prostate-specific antigen (PSA) using microcantilevers. Nat. Biotech. 19, 856-860 (2001).

5. Fritz, J. et al. Translating biomolecular recognition into nanomechanics. Science. 288, 316-318 (2000).

6. Dwyer, D.J., et al. Antibiotic-induced bacterial cell death exhibits physiological and biochemical hallmarks of apoptosis. Mol. Cell. 46, 561-572 (2012).

7. Kohanski, M.A., Dwyer, D.J., Wierzbowski, J., Cottarel, G., \& Collins, J.J. Mistranslation of membrane proteins and two-component system activation trigger antibiotic-mediated cell death. Cell. 135, 679-690 (2008) .

8. Kahne, D. Leimkuhler, C., Wei, L., \& Walsh, C. Glycopeptide and lipoglycopeptide antibiotics. Chem. Revs. 105, 425-448 (2005).

9. Williams, D.H., Maguire, A.J., Tsuzuki, W., \& Westwell, M.S. An analysis of the origins of a cooperative binding energy of dimerization. Science. 280, $711-714$ (1998)

10. Bugg, T.D.H., et al. Molecular-basis for vancomycin resistance in enterococcus faecium BM4147- biosynthesis of a depsipeptide peptidoglycan precursor by vancomycin resistance proteins VanH and VanA. Biochem. 30, 10408-10415 (1991).

11. Neu, H.C. The crisis in antibiotic resistance. Science. 257, 1064-1073 (1992).

12. Walsh, C. Molecular mechanisms that confer antibacterial drug resistance. Nature. 406, $775-781$ (2000).

13. Xu, J. \& Li, H.L. The chemistry of self-assembled long-chain alkanethiol monolayers on gold. J. Colloid Interface Sci. 176, 138-149 (1995).

14. Wink, T., van Zuilen, S.J., Bult, A., \& van Bennekom, W.P. Self-assembled monolayers for biosensors. Analyst. 122, 43-50 (1997).

15. Cho Y.R., Entress R.M. \& Williams, D.H. Synthesis of cell-wall analogues of vancomycin-resistant enterococci using solid phase peptide synthesis. Tetrahedron Lett. 38, 5229-5232 (1997).

16. Prime, K.L. \& Whitesides, G.M. Self-assembled organic monolayers - model systems for studying adsorption of proteins at surfaces. Science. 252, 1164-1167 (1991).

17. Rotschafer, J.C., et al. Pharmacokinetics of Vancomycin: Observations in 28 Patients and Dosage Recommendations. Antimicrob. Agents Chemother. 22, 391-394 (1982).

18. Cooper, M.A., Fiorini, M.T., Abell, C., \& Williams, D.H. Binding of vancomycin group antibiotics to D-alanine and D-lactate presenting selfassembled monolayers. Bioorg. Med. Chem. 8, 2609-2616 (2000).

19. Rao, J., Yan, L., Xu, B., \& Whitesides, G.M. Using surface plasmon resonance to study the binding of vancomycin and its dimer to selfassembled monolayers presenting D-Ala-D-Ala. J. Am. Chem. Soc. 121, 2629-2630 (1999).

20. MacBeath, G. \& Schreiber, S.L. Printing Proteins as Microarrays for High-Throughput Function Determination. Science. 289, 1760-1763 (2000).

21. Baller, M.K., et al. A cantilever array-based artificial nose. Ultramicroscopy. 82, 1-9 (2000).

22. Burg, T.P., et al. Weighing of biomolecules, single cells and single nanoparticles in fluid. Nature. 446, 1066-1069 (2007).

23. Lahiri, J., Isaacs, L., Tien, J., \& Whitesides, G.M. A strategy for the generation of surfaces presenting ligands for studies of binding based on an active ester as a common reactive intermediate: A surface plasmon resonance study. Anal. Chem. 71, 777-790 (1999).

24. Nugaeva, N., et al. Micromechanical cantilever array sensors for selective fungal immobilization and fast growth detection. Biosens. Bioelectron. 21, 849-856 (2005).

25. Von Muhlen, M.G., et al. Label-free biomarker sensing in undiluted serum with suspended microchannel resonators. Anal. Chem. 82, 1905-1910 (2010).

26. M. Godin, M., et al. Using buoyant mass to measure the growth of single cells, Nat. Methods. 7, 387390 (2010).

27. Mertens, J., et al. Detection of gas trace of hydrofluoric acid using microcantilever. Sens. Actuators B Chem. 99, 58-65 (2004).

28. Porter, T.L., et al. A solidstate sensor platform for the detection of hydrogen cyanide gas. Sens. Actuator B Chem. 123, 313-317 (2007). 